\title{
3D Human Body Tracking using Deterministic Temporal Motion Models
}

\author{
Raquel Urtasun and Pascal Fua* \\ Computer Vision Laboratory \\ EPFL \\ CH-1015 Lausanne, Switzerland \\ raquel.urtasun, pascal.fua@epfl.ch
}

\begin{abstract}
There has been much effort invested in increasing the robustness of human body tracking by incorporating motion models. Most approaches are probabilistic in nature and seek to avoid becoming trapped into local minima by considering multiple hypotheses, which typically requires exponentially large amounts of computation as the number of degrees of freedom increases.

By contrast, in this paper, we use temporal motion models based on Principal Component Analysis to formulate the tracking problem as one of minimizing differentiable objective functions. The differential structure of these functions is rich enough to yield good convergence properties using a deterministic optimization scheme at a much reduced computational cost. Furthermore, by using a multi-activity database, we can partially overcome one of the major limitations of approaches that rely on motion models, namely the fact they are limited to one single type of motion.

We will demonstrate the effectiveness of the proposed approach by using it to fit full-body models to stereo data of people walking and running and whose quality is too low to yield satisfactory results without motion models.
\end{abstract}

\section{Introduction}

In recent years, much work has been devoted to increasing the robustness of people tracking algorithms by introducing motion models. Most approaches rely on probabilistic methods, such as the popular CONDENSATION algorithm [1,2], to perform the tracking. While effective, such probabilistic approaches require exponentially large amounts of computation as the number of degrees of freedom in the model increases, and can easily become trapped into local minima unless great care is taken to avoid them $[3,4,5,6]$.

By contrast, in this paper, we use temporal motion models based on Principal Component Analysis (PCA) and inspired by those proposed in $[7,8,9]$ to formulate the tracking problem as one of minimizing differentiable objective

\footnotetext{
* This work was supported in part by the Swiss National Science Foundation.
} 
functions. Our experiments show that the differential structure of these objective functions is rich enough to take advantage of standard deterministic optimization methods [10], whose computational requirements are much smaller than those of probabilistic ones and can nevertheless yield very good results even in difficult situations. Furthermore, in practice, we could combine both kinds of approaches [5].

We will further argue that we can partially overcome one of the major limitations of approaches that rely on motion-models, namely that they limit the algorithms to the particular class of motion from which the models have been created. This is achieved by performing PCA on motion databases that contain multiple classes of motions as opposed to a single one, which yields a decomposition in which the first few components can be used to classify the motion and can evolve during tracking to model the transition from one kind of motion to another.

We will demonstrate the effectiveness of the proposed approach by using it to fit full-body models to stereo data of people walking and running and whose quality is too low to yield satisfactory results without models. This stereo data simply provides us with a convenient way to show that this approach performs well on real data. However, any motion tracking algorithm that relies on minimizing an objective function is amenable to the treatment we propose. We therefore view the contribution of this paper as the proposed formulation that produces results using a deterministic, as opposed to probabilistic optimization method, which yields good performance at a reduced computational cost.

In the remainder of this paper, we first discuss related approaches and our approach to body and motion modeling. We then introduce our deterministic optimization scheme and show its effectiveness using real data.

\section{Related Work}

Modeling the human body and its motion is attracting enormous interest in the Computer Vision community, as attested by recent and lengthy surveys [11,12]. However, existing techniques remain fairly brittle for many reasons: Humans have a complex articulated geometry overlaid with deformable tissues, skin and loosely-attached clothing. They move constantly, and their motion is often rapid, complex and self-occluding. Furthermore, the $3-\mathrm{D}$ body pose is only partially recoverable from its projection in one single image. Reliable $3-\mathrm{D}$ motion analysis therefore requires reliable tracking across frames, which is difficult because of the poor quality of image-data and frequent occlusions.

When a person is known a priori to be performing a given activity, such as walking or running, an effective means to constrain the search and increase robustness is to introduce a motion model. Of particular interest to us are models that represent motion vectors as linear sums of principal components and have become widely accepted in the Computer Animation community as providing realistic results $[13,14,15]$. The PCA components are computed by capturing as many people as possible performing a specific activity, for example by means of an optical motion capture system, representing each motion as a temporally 
quantized vector of joint angles, and performing a Principal Component Analysis on the resulting set of vectors.

In practice, the position of a person, or body pose, in a given image frame can be defined by the position and orientation of a root node and a vector of joint angles. A motion can then be represented by an angular motion vector, that is a set of such joint angle vectors measured at regularly sampled intervals. Given a large enough database of motion vectors for different motion classes and the corresponding principal components $\Theta j, 1 \leq j \leq m$, at a given time $t$, the joint angle vector $\Theta\left(\mu_{t}\right)$ can then be written as

$$
\Theta\left(\mu_{t}\right)=\Theta_{0}\left(\mu_{t}\right)+\sum_{j=1}^{m} \alpha_{j} \Theta_{j}\left(\mu_{t}\right) \quad \text { with } 0 \leq \mu_{t} \leq 1,
$$

where $\mu_{t}$ is a normalized temporal variable that indicates to what stage of the motion the pose corresponds, $\Theta_{0}$ represents an average motion, and the $\alpha_{j}$ are scalar coefficients. In short, the vector $\left(\mu_{t}, \alpha_{1}, \ldots, \alpha_{m}\right)$, where $m$ is much smaller than the number of joint angles, can be used as the state vector that completely describes the body pose. Recovering this pose then amounts to minimizing an image-based objective function $F$ with respect to this more compact representation, and can be expected to be much more robust than minimizing it with respect to the full set of joint angles.

This representation has already been successfully used in our community, but almost always in a statistical context $[7,8,9]$ and without exploiting the fact that $F$ is easily differentiable with respect to $\mu_{t}$ and the $\alpha_{j}$ coefficients of Eq. 1. Here, we propose to use this fact to formulate the fitting problem as a traditional optimization problem with respect to the $\left(\mu_{t}, \alpha_{1}, \ldots, \alpha_{N}\right)$ state vector. Instead of generating many "particles" by randomly choosing values for the $\alpha_{j}$, we will compute the Jacobian of $F$ and use it in conjunction with standard least-squares techniques [16]. Our deterministic approach to motion tracking is therefore related to an earlier technique [17] that also uses PCA to model the set of 2-D flow vectors that can be seen in video-sequences of a walking subject and to recognize specific $2-\mathrm{D}$ poses without requiring a probabilistic framework. However, this approach relies on an initial segmentation of the body parts and is viewpoint dependent. By contrast, we fit a global 3-D model to the whole body, which lets us fit over a whole sequence and recover accurate $3-\mathrm{D}$ poses.

\section{Models}

In this section, we introduce the models we use to describe both body pose and shape at a given time as well as its motion over time.

\subsection{Body Model}

In earlier work [18], we have developed a body-modeling framework that relies on attaching implicit surfaces, also known as soft objects, to an articulated skeleton. Each primitive defines a field function and the skin is taken to be a level set of the 
sum of these fields, as shown in Fig. 1a. Defining surfaces in this manner lets us define a distance function of data points to the model that is differentiable. We will take advantage of this to implement our minimization scheme, as discussed in Section 4.
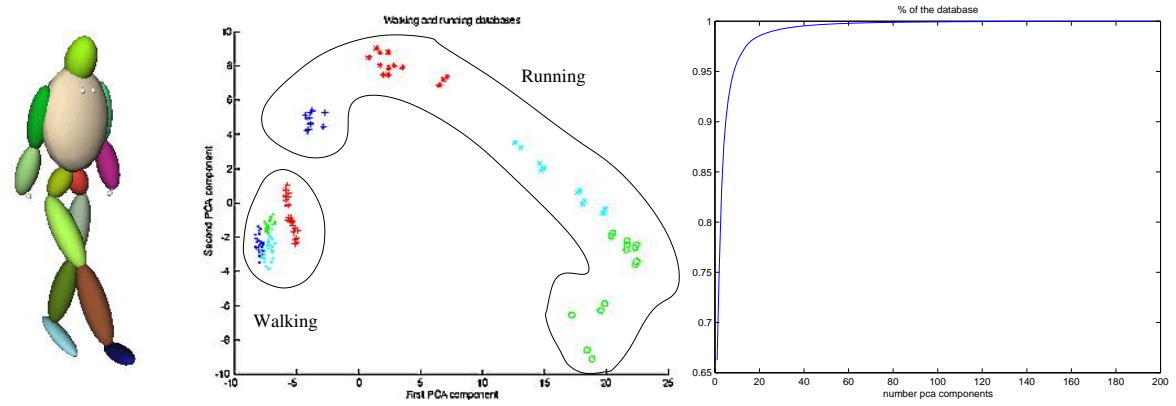

Fig. 1. Shape and motion models. a) Volumetric primitives attached to an articulated skeleton. b) First two PCA components for 4 different captures of 4 subjects walking at speeds varying from 3 to $7 \mathrm{~km} / \mathrm{h}$, and running at speeds ranging from 6 to $12 \mathrm{~km} / \mathrm{h}$. The data corresponding to different subjects is shown in different styles. c) Percentage of the database that can be generated with a given number of eigenvectors.

As in Section 2, let us assume that, at a given time, the pose of the skeleton is entirely characterized by the global position and orientation $G$ of a root node and a set of joint angles $\Theta$. To avoid undue blending of primitives, the body is divided into several body parts. Each body part $b$ includes $n_{b}$ ellipsoidal primitives attached to the skeleton. To each primitive is associated a field function $f_{i}$ of the form $f_{i}(G, \Theta, X)=b_{i} \exp \left(-a_{i} d_{i}(G, \Theta, X)\right)$, where $X$ is a $3-\mathrm{D}$ point, $a_{i}, b_{i}$ are constant values, and $d_{i}$ is the algebraic distance to this ellipsoid. The complete field function for body part $b$ is taken to be

$$
f^{b}(G, \Theta, X)=\sum_{i=1}^{n_{b}} f_{i}(X, G, \Theta)
$$

and the skin is the set $\mathcal{S}(G, \Theta)=\bigcup_{b}\left\{X \in \Re^{3} \mid f^{b}(G, \Theta, X)=C\right\}$, where $C$ is a constant. A point $X$ is said attached to body part $b$ if

$$
f^{b}(G, \Theta, X)=\min _{1 \leq i \leq B}\left|f^{i}(G, \Theta, X)-C\right|
$$

Fitting the model to stereo-data acquired at time $t$ then amounts to minimizing

$$
F_{t}\left(G_{t}, \Theta_{t}\right)=\sum_{b=1}^{B} \sum_{X_{t} \in b}\left(f^{b}\left(G_{t}, \Theta_{t}, X_{t}\right)-C\right)^{2},
$$

where the $X_{t}$ are the 3-D points derived from the data, each one being attached to one of the $B$ body parts. Note that $F_{t}$ is a differentiable function of the global position $G_{t}$ and of the joint angles in $\Theta_{t}$ and that its derivatives can be computed fast [18]. 


\subsection{Motion Models}

To create a motion database, we used a Vicon ${ }^{t m}$ optical motion capture system and a treadmill to capture 4 people, 2 men and 2 women,

- walking at 9 different speeds ranging from 3 to $7 \mathrm{~km} / \mathrm{h}$, by increments of 0.5 $\mathrm{km} / \mathrm{h}$;

- running at 7 different speeds ranging from 6 to $12 \mathrm{~km} / \mathrm{h}$, by increments of $1.0 \mathrm{~km} / \mathrm{h}$.

The data was then segmented into cycles and normalized so that each one is represented by the same number of samples. To this end, spherical interpolation in quaternion space was used because it is the space in which a distance measuring the proximity of two orientations can be naturally defined. It therefore lets us interpolate with a meaningful angular velocity measure on an optimal path splining among orientation key frames [19]. Since people never perform the same motion twice in exactly the same fashion, we included in the database four walking or running cycles for each person and speed. The mean motion of the set of examples was subtracted and the $M$ eigenvectors of Eq. 1 were obtained by SVD. Retaining only $m \leq M$ eigenvectors, gave us a reduced base of the most significant subspace of the motion space, that is the one that contains $\sigma \%$ of the database, where $\lambda_{i}$ is the i-th bigger eigenvalue.

$$
\sigma=\frac{\sum_{i=1}^{m} \lambda_{i}}{\sum_{i=1}^{M} \lambda_{i}}
$$

In our experiments, we chose $\sigma=0.9$, which means that for the multi-activity database we need only 5 out of 256 coefficients, which corresponds to the total number of examples in the database. In Fig. 1c we display $\sigma$ as a function of the number of eigenvectors. The same method was used for the walking and running databases independently. The estimation problem is thus reduced from the $\simeq 80$ degrees of freedom for the 28 joints in our body model at each time step, to 5 coefficients plus the time.

Fig. 1b shows the first two PCA components of the original examples used to create the joint walking and running database. The two activities produce separate clusters. The walking components appear on the left of the plot and form a relatively dense set. By contrast, running components are sparser because inter-subject variation is larger, indicating that more examples are required for a complete database.

Note that varying only the first two components along the curve corresponding to the path from one subset to another, yields very natural transitions between walking and running motions.

\section{Deterministic Approach to Tracking}

In this section we introduce our deterministic approach to tracking that relies on describing motion as a linear combination of the motion eigenvectors of Section 3.2 and choosing optimal weights for these vectors. As before, we represent 
the angular component of motion $\Theta$ as $\Theta=\Theta_{0}+\sum_{i=1}^{m} \alpha_{i} \Theta_{i}$, where $\Theta_{0}$ is the average motion and the $\Theta_{i}$ are the eigenvectors of Section 3.2. Evaluating $\Theta$ at a particular time $\mu_{t}$ yields the pose

$$
\Theta\left(\mu_{t}\right)=\Theta_{0}\left(\mu_{t}\right)+\sum_{i=1}^{m} \alpha_{i} \Theta_{i}\left(\mu_{t}\right)=\left[\theta^{1}\left(\mu_{t}\right), \cdots, \theta^{\text {ndof }}\left(\mu_{t}\right)\right]^{T}
$$

where the $\theta^{j}$ are the actual joint angles at time $\mu_{t}$ for the $n d o f$ degrees of freedom of the body model we use.

Note that the complete motion is described not only by the angular motion discussed above, but also by the motion $G_{t}$ of the root body model node with respect to which the angles are expressed. This adds six degrees of freedom to our model, which are not represented at all in our motion database since the data was acquired on a treadmill on which the subjects were forced to walk straight. Furthermore, even if the global motion had been acquired, it would make no sense to include it in the database because similar motions would then have been considered as different just because of the orientation or position of the body.

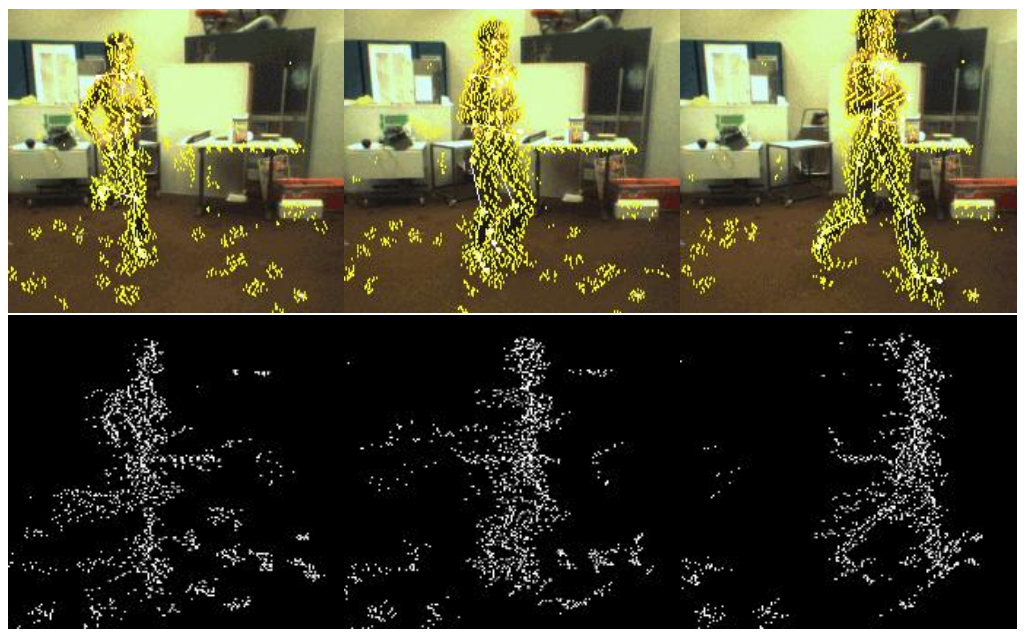

Fig. 2. Input stereo data. Top row: First image of a synchronized trinocular video sequence at three different times. The $3-\mathrm{D}$ points computed by the Digiclops ${ }^{t m}$ system are reprojected onto the images. Bottom row: Side views of these $3-D$ points. Note that they are very noisy and lack depth because of the low quality of the video sequence.

Let us assume that we have acquired image data, which here is the stereo data depicted by Fig. 2, but could just as well be anything else, in $T$ consecutive frames. Our goal is to recover the motion by minimizing an objective function $F$ over all frames and, therefore, fitting the model to the image data. Tracking is achieved in two main steps. First the global motion $G_{t}$ is recovered in a recursive way. Results from frame $t$ are used as initialization for frame $t+1$. We initialize using the average motion $\Theta_{0}$, positioning the global motion for the first 
frame by hand, where the time $\mu_{t}, 1 \leq t \leq T$, is a linear interpolation between initial values $\mu_{1}$ and $\mu_{T}$ for the first and last frames. For each frame we minimize $F_{t}\left(G_{t}, \Theta_{0}\left(\mu_{t}, \alpha_{i}\right)\right)$ with respect to $G=G\left(t_{x}, t_{y}, t_{z}, \theta_{x}, \theta_{y}, \theta_{z}\right)$, where $F_{t}$ is defined in equation 4 . Given this global motion estimate, we then fit the data over all frames simultaneously by minimizing $F$ with respect to the $\mu_{t}, \alpha_{i}$ and $G_{t}$ :

$$
F=\sum_{1 \leq t \leq T} F_{t}\left(G_{t}, \Theta\left(\mu_{t}, \alpha_{i}\right)\right),
$$

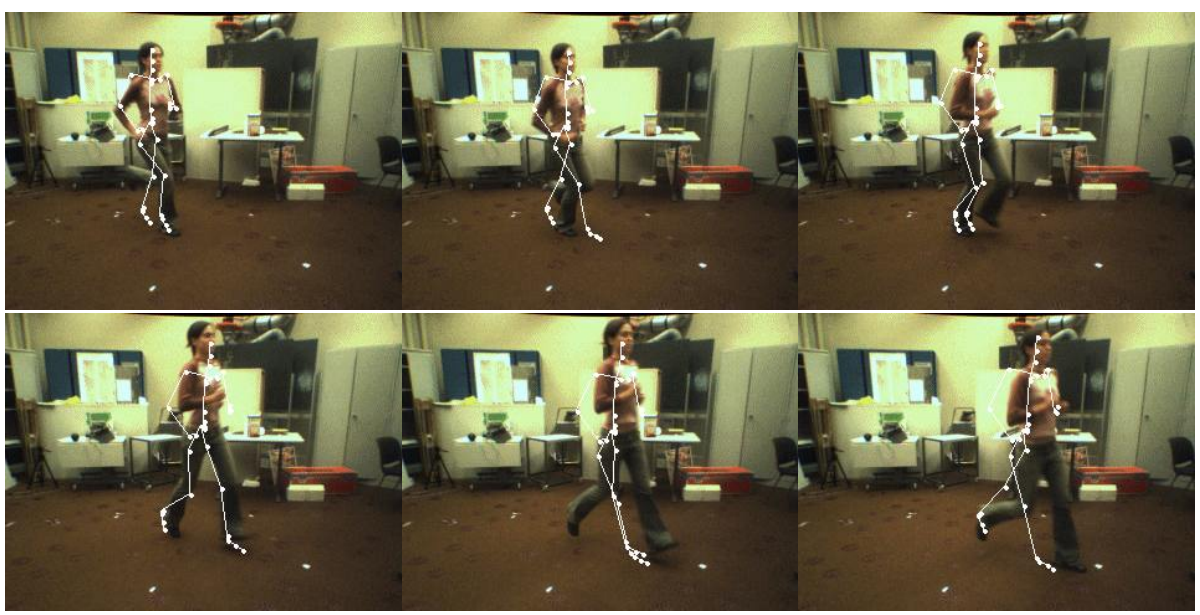

Fig. 3. Tracking without a motion model. Given the low framerate, motion between frames is large enough to provoke erroneous attachments of data points to body parts and, as a consequence, very poor fitting behavior. The whole sequence is shown.

In the remainder of this Section, for comparison purposes, we first show the result of fitting the stereo data we use without using motion models. We then introduce in more detail our approach to enforcing the motion models, with or without assuming that the style remains constant. Finally, we discuss the computational requirements of our scheme and contrast them with those of more traditional probabilistic approaches.

\subsection{Tracking without Motion Models}

In this paper we use stereo data acquired using a Digiclops ${ }^{t m}$ operating at a $640 \times 480$ resolution and a $14 \mathrm{~Hz}$ framerate, which is relatively slow when it comes to capturing a running motion. The quality of the data is poor for several reasons. First, to avoid motion blur, we had to use a high shutter speed that reduces exposure too much. Second, because the camera is fixed and the subject must remain within the capture volume, she appears to be very small at the beginning of the sequence. As a result the data of Fig. 2 is very noisy and lacks both resolution and depth.

To establish a baseline, in Fig. 3, we show the unsatisfactory result of fitting our model to this data without using motion models, that is by minimizing the 
objective function of Eq. 4 in each frame separately. We simply use the recovered pose in frame $t-1$ as the starting point in frame $t$.

Careful analysis shows that tracking fails chiefly due to the low framerate, as the interframe motion is too large. This prevents the process of "attaching" data points to body parts discussed in Section 3.1 from functioning properly. In the fifth image of Fig. 3, both legs end up being "attracted" to the same data points.

\subsection{Tracking a Steady Motion}

To remedy the problems discussed above, we can first assume that the motion is steady over $T$ data frames and, therefore, that the $\alpha_{i}$ coefficients of Eq. 6 are invariant. The motion state vector is taken to be

$$
\phi=[\vec{\mu}, \vec{\alpha}]=\left[\mu_{1}, \ldots, \mu_{T}, \alpha_{1}, \ldots, \alpha_{m}\right]
$$

To effectively minimize the objective function $F$ of Eq. 7 using a standard leastsquares technique [16], we need to evaluate its Jacobian. Bearing in mind that the derivatives of $F$ with respect to the individual joints angles $\frac{\partial F}{\partial \theta_{j}}$ can be easily computed [18], this can be readily done as follows:

$$
\frac{\partial F}{\partial \alpha_{i}}=\sum_{j=1}^{n d o f} \frac{\partial \theta_{j}}{\partial \alpha_{i}} \cdot \frac{\partial F}{\partial \theta_{j}}, \quad \frac{\partial F}{\partial \mu_{t}}=\sum_{j=1}^{n d o f} \frac{\partial \theta_{j}}{\partial \mu_{t}} \cdot \frac{\partial F}{\partial \theta_{j}} .
$$

Because the $\theta_{j}$ are linear combinations of the $\Theta_{i}$ eigenvectors, $\frac{\partial \theta_{j}}{\partial \alpha_{i}}$ is simply the $\Theta_{i j}$, the $j$ th coordinate of $\Theta_{i}$. Similarly, we can write

$$
\frac{\partial \theta_{j}}{\partial \mu_{t}}=\sum_{i=1}^{m} \alpha_{i} \frac{\partial \Theta_{i j}}{\partial \mu_{t}}
$$

where the $\frac{\partial \Theta_{i j}}{\partial \mu_{t}}$ can be evaluated using finite differences and stored when building the motion database, as shown in Fig. 4.

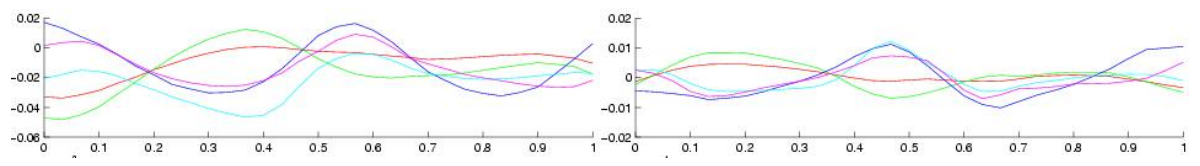

Fig. 4. Motion vector and its temporal derivatives. Left: First 5 eigenvectors for the flexion-extension in the sagittal plane of the left knee. Right: Temporal derivatives $\frac{\partial \Theta_{i j}}{\partial \mu_{t}}$.

Figure 5 depicts results on a walking sequence performed by a subject whose motion was captured when building the database. Note that the legs are correctly positioned. The errors in the upper-body are due to the noisyness of the stereo cloud. 

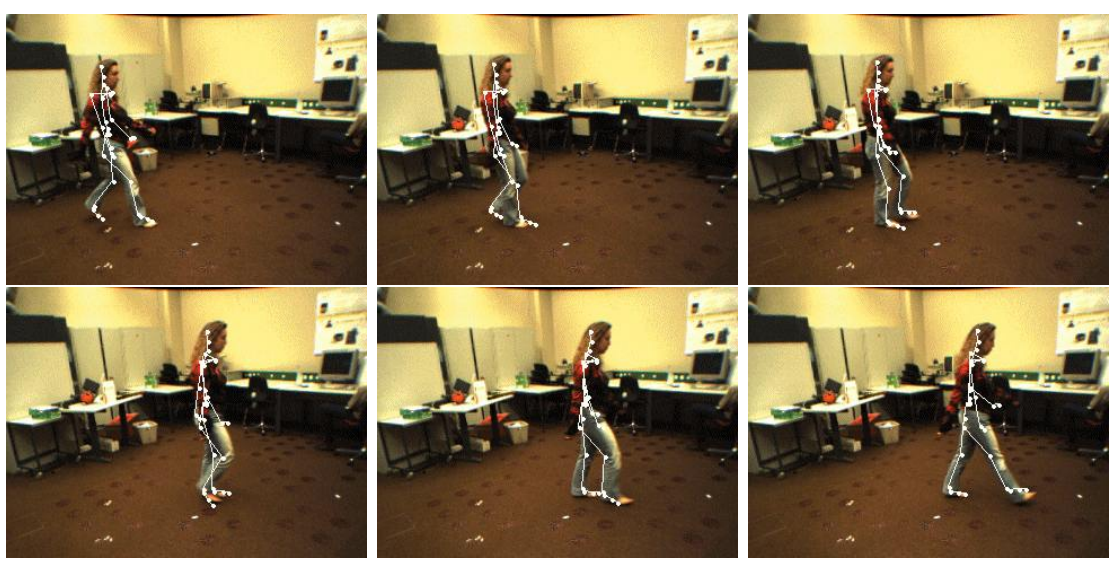

Fig. 5. Using low resolution stereo data to track a woman whose motion was recorded in the database. The recovered skeleton poses are overlaid in white. The legs are correctly positioned.

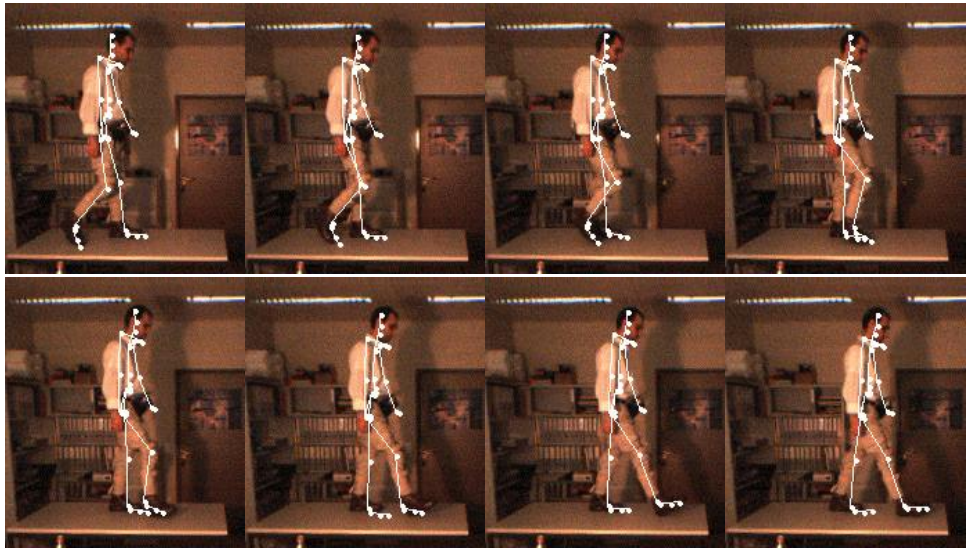

Fig. 6. Tracking a walking motion assuming a constant style. The legs are correctly positioned.

Fig. 6 displays the results on a walking sequence performed by a subject who was not recorded when building the database. To validate our results, he is wearing four gyroscopes on his legs, one for each sagittal rotation of the hip and knee joints. The angular speeds they measure are used solely for comparison purposes and we show their integrated values in Fig. 7. We overlay on the corresponding plots the values recovered by our tracker. Note that they are very close, even though the left leg is severely occluded.

Fig. 8 depicts results on the running sequence of Fig. 2 using the running database of Section 3.2, which are much better than those of Fig. 3. The pose of the legs is now correctly recovered, except the one of the left leg in the first frame. This is due in part to the fact that the database was acquired using a treadmill and is therefore too sparse to model a motion in which the leg is raised 

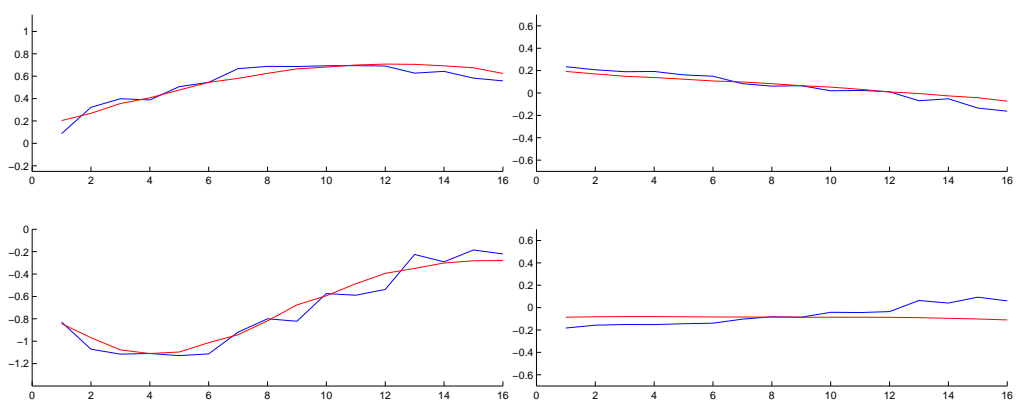

Fig. 7. Comparing recovered rotation angles using visual tracking (solid curve), and by integrating gyroscopic data (smooth curve) for the walk of Fig. 6. Left column: Right hip and knee sagittal rotations. Right Column: Same thing for the left leg. Note that both curves are very close in all plots, even though the left leg is severely occluded.

that high, and in part to the fact that the motion is not truly steady. We address these issues below.

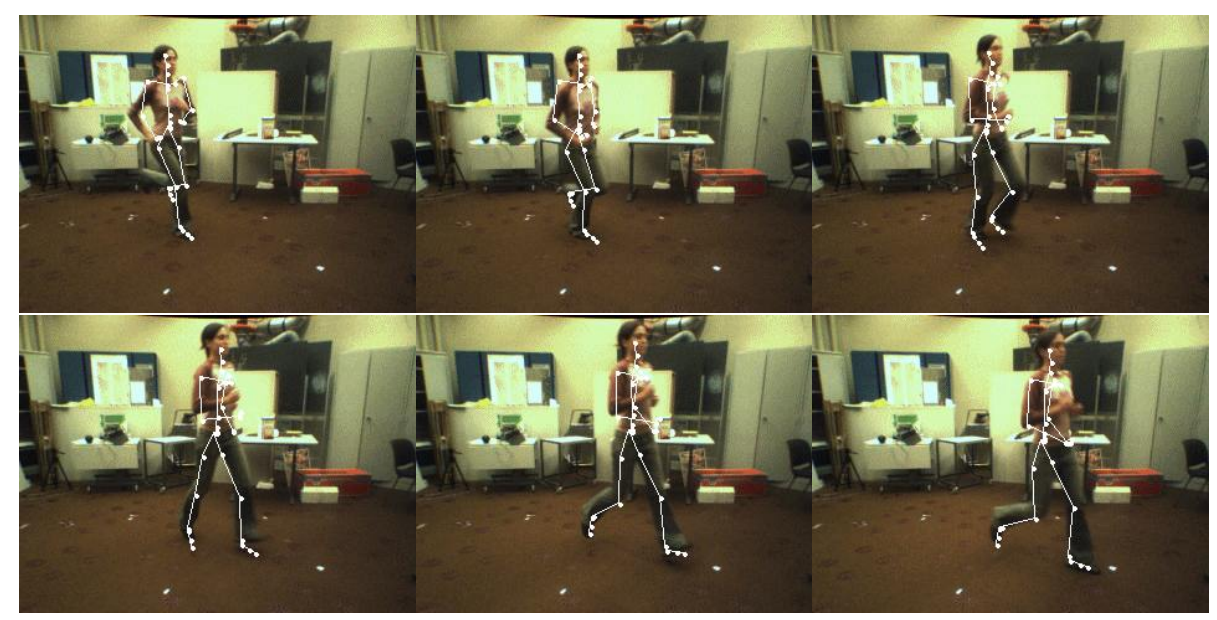

Fig. 8. Tracking a running motion assuming a constant style. The legs are correctly positioned except the left one in the first frame.

\subsection{Tracking a Variable Style and Speed Motion}

In the sequences shown in this paper, speed and style are not truly constant. Because of space constraints, the subject starts, accelerates and stops over a short distance. This is true for walking and running, and even more so for transitions from one to the other. Using a single set of $\alpha_{i}$ parameters for the whole sequence as in Section 4.2 therefore overconstrains the problem. We relax these constraints by introducing a set of $\alpha_{i}$ per frame or per set of frames and the state vector then becomes:

$$
\phi=\phi\left(\vec{\mu}, \overrightarrow{\alpha^{1}}, \ldots, \overrightarrow{\alpha^{T}}\right) \text { where } \overrightarrow{\alpha^{i}}=\left(\alpha_{1}^{i}, \ldots, \alpha_{m}^{i}\right) .
$$


Improved tracking results from the running sequence of Fig. 2 are shown in Fig. 9. The system now has enough freedom to raise the leg in the first frame while still positioning the legs correctly everywhere else. Upper body tracking remains relatively imprecise because average errors in the stereo data are larger than the distance between torso and arms. Improving this would require the use of additional information, such as silhouette information, which could easily be done within the proposed framework. Similar results for walking are shown in Fig. 10. Small errors in foot positioning are due to the fact that ankle flexion has not been recorded in the motion database.

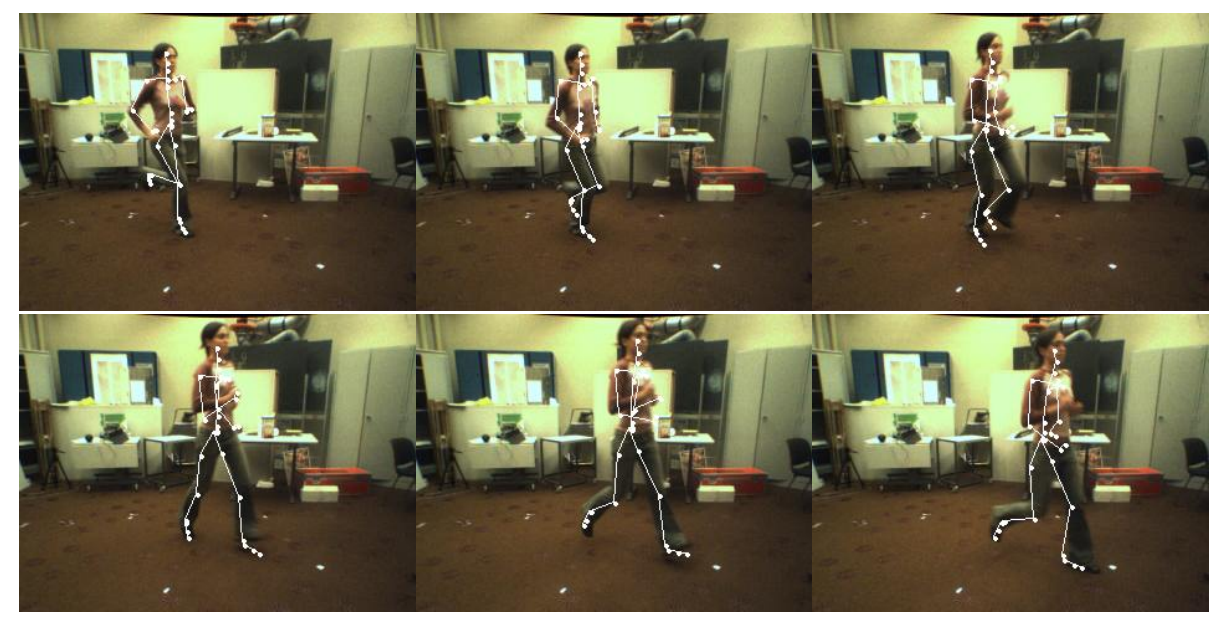

Fig. 9. Tracking a running motion while allowing the style to vary. The legs are now correctly positioned in the whole sequence.

Having a set of PCA parameters per frame gives the system the freedom to automatically evolve from one activity to another. To demonstrate this, in Fig. 11, we use our full motion database to track a transition from walking to running. In the first few frames the subject is walking, then for a couple of frames she performs the transition and runs for the rest of the sequence. The arms are not tracked because we focus on estimating the motion parameters of the lower body only. Here again, the legs are successfully tracked with small errors in foot positioning that are due to the fact that ankle flexion is not part of the motion database.

\subsection{Computational Requirements}

Probabilistic approaches such as the one of [8] rely on randomly generating "particles" and evaluating their fitness. Assuming the cost of creating the particles to be negligible, the main cost of each iteration comes from evaluating an objective function, such as the function $F$ of Eq. 7 for each particle. In the classical implementation of the condensation, where the state vector has ndofs degrees of freedom, the cost is therefore in the order of $\mathcal{O}$ (npart(ndofs)) times the cost 


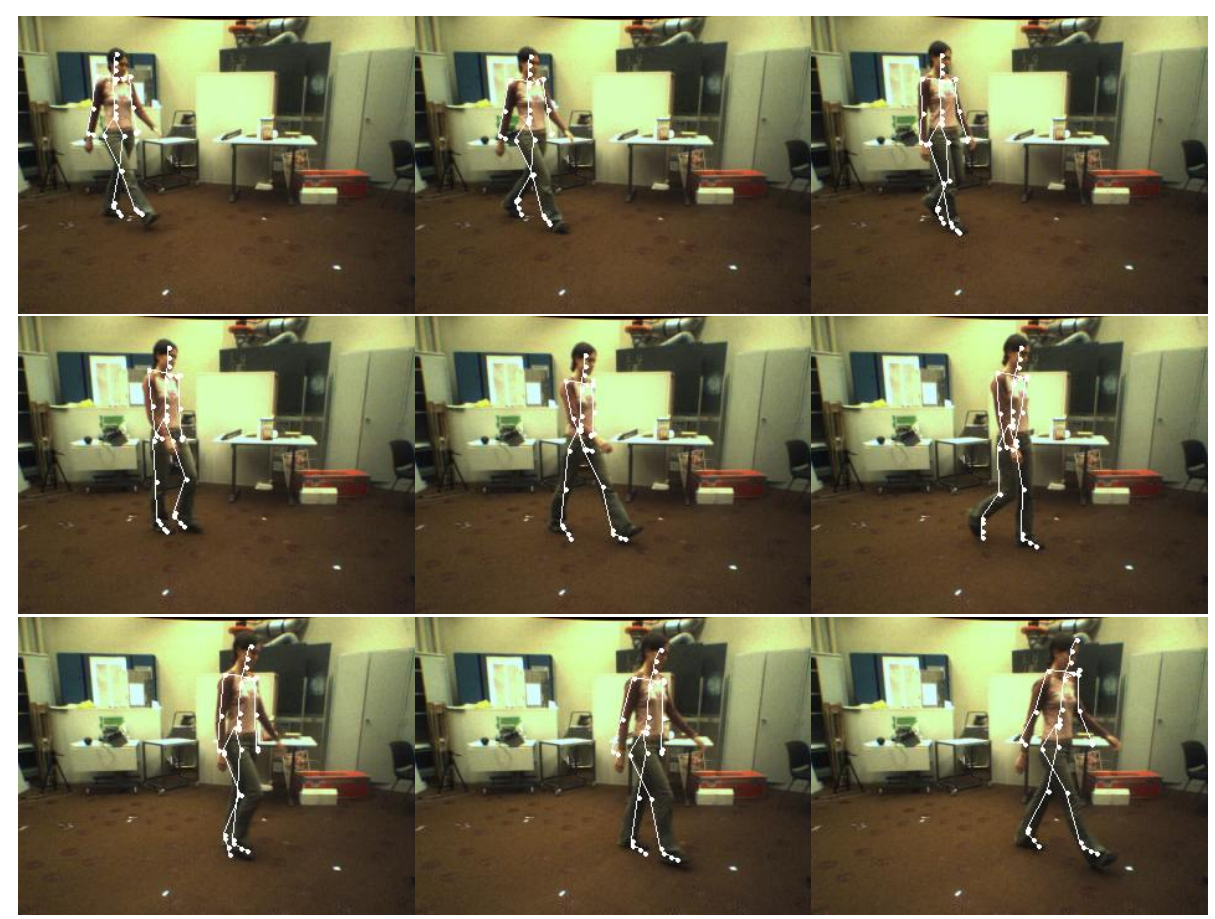

Fig. 10. Tracking a walking motion while allowing the style to vary. The sequence has a total of 18 frames, we show one in two.

of computing $F$, where npart is the number of particles, which tends to grow very fast if good convergence properties are to be achieved. On the other hand, if we use our motion models to perform the condensation, the cost is of the order of $\mathcal{O}(n \operatorname{part}(n))$, which also grows fast with $n$, the state's vector dimension.

By contrast, the main cost of each iteration of our optimization scheme comes from evaluating $F$ and its Jacobian, which is of course more expensive than evaluating $F$ alone. However, through careful implementation, we have found that it can be done at a cost in the order of $\mathcal{O}(\lg (n d o f))$ times the cost of computing $F$ alone, since evaluating $F$ and its derivatives for the ndof degrees of freedom in the body model involves many similar computations, and computing $\frac{\partial F}{\partial \theta_{j}}$ once per iteration is what is costly. It took less than 15 iterations to achieve convergence. As a consequence, the cost of the methods of Section 4.2 and 4.3 are of the same order and smaller than the probabilistic approach.

\section{Conclusion}

We have presented an approach using motion models that allows us to formulate the tracking problem as one of minimizing a differential objective function with respect to relatively few parameters. We take them to be the first few coefficients of the principal components of the joint angle space for motions captured using an optical motion capture device. 


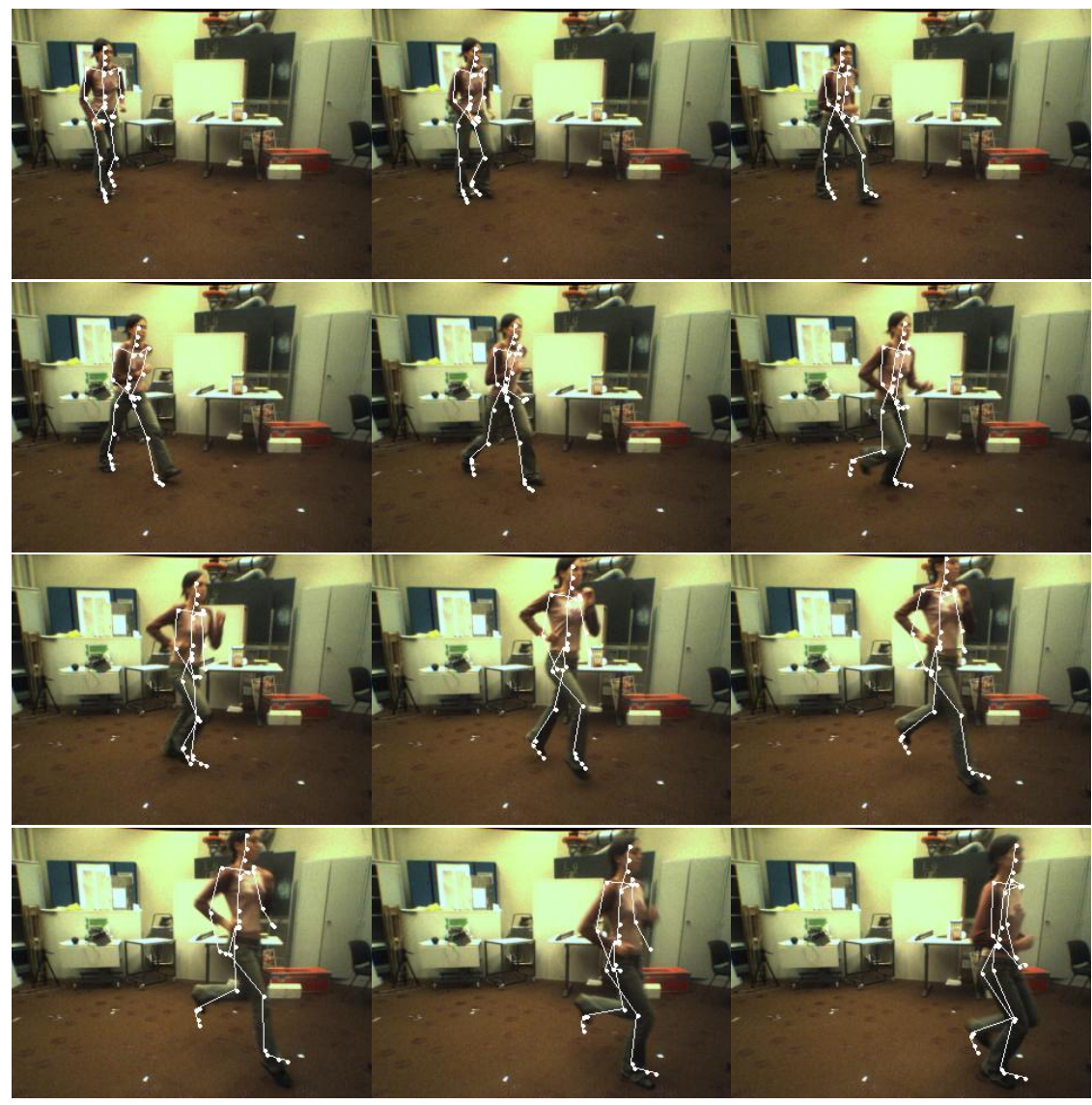

Fig. 11. Tracking the transition between walking and running. In the first four frames the subject is running. The transition occurs in the following three frames and the sequence ends with running. The whole sequence is shown.

Using walking and running as examples, we have shown that this representation, while having a fairly low dimension, nevertheless has a rich enough differential structure to yield good performance at a low computational cost. It also has the ability to capture the transition from one motion to another.

We have demonstrated that our approach can simultaneously handle two different activities. Our method seems perfectly adapted to 3-D analysis of sport activities such as a golf swing or a tennis serve. The same can be said of capturing the motion of orthopedic patients when they are asked to perform a paticular routine designed to evaluate their conditions. Applying our method to such activities will be a subject for future research.

Currently, the major limitation comes from the small size of the database we use, which we will endeavor to complete. This should allow us to precisely track a wider range of styles, perhaps at the cost of adding some regularization constraints that we presently do not need. We also plan to add additional motion types, such as jumping, for which motion capture data is fairly easy to acquire. 
In the current database, the samples corresponding to different people tend to cluster. If this remains true when the database is completed, this may become a promising approach not only for tracking but also for recognition.

\section{References}

1. Isard., M., Blake, A.: CONDENSATION - conditional density propagation for visual tracking. International Journal of Computer Vision 29 (1998) 5-28

2. Deutscher, J., Blake, A., Reid, I.: Articulated Body Motion Capture by Annealed Particle Filtering. In: CVPR, Hilton Head Island, SC (2000)

3. Davison, A.J., Deutscher, J., Reid, I.D.: Markerless motion capture of complex fullbody movement for character animation. In: Eurographics Workshop on Computer Animation and Simulation, Springer-Verlag LNCS (2001)

4. Choo, K., Fleet, D.: People tracking using hybrid monte carlo filtering. In: International Conference on Computer Vision, Vancouver, Canada (2001)

5. Sminchisescu, C., Triggs, B.: Covariance Scaled Sampling for Monocular 3D Body Tracking. In: Conference on Computer Vision and Pattern Recognition, Hawaii (2001)

6. Sminchisescu, C., Triggs, B.: Kinematic Jump Processes for Monocular 3D Human Tracking. In: Conference on Computer Vision and Pattern Recognition, Madison, WI (2003)

7. Sidenbladh, H., Black, M.J., Fleet, D.J.: Stochastic tracking of 3D human figures using 2D image motion. In: European Conference on Computer Vision. (2000)

8. Sidenbladh, H., Black, M.J., Sigal, L.: Implicit Probabilistic Models of Human Motion for Synthesis and Tracking. In: European Conference on Computer Vision, Copenhagen, Denmark (2002)

9. Ormoneit, D., Sidenbladh, H., Black, M.J., Hastie, T.: Learning and tracking cyclic human motion. In: Advances in Neural Information Processing Systems 13. (2001) 894-900

10. Bregler, C., Malik, J.: Tracking People with Twists and Exponential Maps. In: Conference on Computer Vision and Pattern Recognition, Santa Barbara, CA (1998)

11. Gavrila, D.: The Visual Analysis of Human Movement: A Survey. Computer Vision and Image Understanding $\mathbf{7 3}$ (1999)

12. Moeslund, T., Granum, E.: A Survey of Computer Vision-Based Human Motion Capture. Computer Vision and Image Understanding 81 (2001)

13. Alexa, M., Mueller, W.: Representing animations by principal components. In: Eurographics. Volume 19. (2000)

14. Brand, M., Hertzmann, A.: Style Machines. Computer Graphics, SIGGRAPH Proceedings (2000) 183-192

15. Blanz, V., Basso, C., Poggio, T., Vetter, T.: Reanimating Faces in Images and Video. In: Eurographics, Granada, Spain (2003)

16. Press, W., Flannery, B., Teukolsky, S., Vetterling, W.: Numerical Recipes, the Art of Scientific Computing. Cambridge U. Press, Cambridge, MA (1992)

17. Yacoob, Y., Davis, L.S.: Learned Models for Estimation of Rigid and Articulated Human Motion from Stationary or Moving Camera. International Journal of Computer Vision 36 (2000) 5-30

18. Plänkers, R., Fua, P.: Articulated Soft Objects for Multi-View Shape and Motion Capture. IEEE Transactions on Pattern Analysis and Machine Intelligence (2003)

19. Shoemake, K.: Animating Rotation with Quaternion Curves. Computer Graphics, SIGGRAPH Proceedings 19 (1985) 245-254 